\title{
Cooperation with Complex Research Protocols: The Use of Global Positioning Systems with Cognitively Impaired Elders - A Preliminary Report
}

\author{
Gail K. Auslander ${ }^{1}$, Shirli Werner ${ }^{* 1}$, Noam Shoval ${ }^{2}$, Michal Isaacson ${ }^{2}$, Ruth Landau ${ }^{1}$ and Jeremia Heinik ${ }^{3}$
}

${ }^{1}$ Paul Baerwald School of Social Work and Social Welfare, The Hebrew University of Jerusalem, Mount Scopus, Jerusalem, 91905, Israel; ' Department of Geography, The Hebrew University of Jerusalem, Mount Scopus, Jerusalem, 91905, Israel; ${ }^{3}$ Margoletz Psychogeriatric Center, Tel-Aviv Sourasky Medical Center, 6 Weizman Street, Tel-Aviv 64239, Israel and Sackler Faculty of Medicine, Tel Aviv University, Tel-Aviv, Israel

\begin{abstract}
Recently developed tracking technologies may be useful in managing mobility problems among elders with cognitive impairment. For clinical and research purposes it is critical to assess research participants' cooperation with the tracking protocol. The aim of the current study was to assess the ability of older adults with various levels of cognitive impairment to participate in GPS-based studies. Fifty-three participants aged $63+$ and their study partners were interviewed. Participants were tracked for four weeks, 24 hours a day, using a location kit combining Global Positioning System with Radio Frequency Identification technology. Participants' cooperation was associated with positive affect $(\mathrm{r}=$ $0.35, \mathrm{p}<.01)$, life satisfaction and study partner's older age $(\mathrm{r}=0.37, \mathrm{p}<.01)$. Advanced tracking technologies offer a feasible method of measuring outdoor mobility behavior of older adults. This preliminary study sets the stage for further data collection via innovative technologies and design interventions to manage mobility problems.
\end{abstract}

Keywords: Alzheimer, compliance, dementia, participation, wandering.

\section{INTRODUCTION}

As the world's population ages, cognitive impairment related to dementia has become more prevalent. A recent consensus study [1] reported a world prevalence of 24.3 million people with dementia in 2001, for a rate of $3.9 \%$ among those aged $\geq 60$. That rate is expected to double every 20 years. Dementia-related disorders constitute a serious threat to the health and well-being of older adults. One of the more common behavioral manifestations of these disorders is problems with out-of-home mobility [2], particularly wandering behavior, estimated at $20-25 \%$ of communitydwelling dementia patients [3].

Outdoor mobility problems affect the family as well as the patient. Disturbing behaviors have been found to be related to higher caregiving burden and depression [4]. Wandering is the most frequently mentioned, most problematic, and least manageable behavior, and is a key factor in the decision to institutionalize older people [5]. These individuals may benefit from different types of interventions [6], including the use of advanced tracking technologies, as described in the current study.

In the past, efforts to assess mobility behavior of people with dementia relied on time-space diaries and observations of caregivers [7]. In recent years sophisticated tracking technologies have been developed that may be useful in measuring and managing mobility and wandering problems and promote safe walking. For example, one recent study has

*Address correspondence to this author at the Paul Baerwald School of Social Work and Social Welfare, The Hebrew University of Jerusalem, Mount Scopus, Jersalem, 91905, Israel; Tel: +9722 5881377; Fax: +9722 5883927; E-mail: shirlior@mscc.huji.ac.il aimed to create acceptable and effective prototype technologies to facilitate independence for people with dementia [8]. The use of electronic tracking devices may reduce the distress and burden of family caregivers and allow them to continue caring for the elder in the community.

One sophisticated tracking technology that has been advanced in recent years is GPS (Global-Positioning Systems)based tracking. Such devices are employed in the SenTra project, a five-year bi-national collaboration between Israeli and German researchers focusing on the use of GPS -based tracking technology to examine out-of-home mobility of older persons with various levels of cognitive decline. SenTra is an interdisciplinary project involving researchers from geography, social work, gerontology, psychology, and medicine. In the SenTra project activity patterns of cognitively impaired persons as well as unimpaired controls are assessed over a period of one month, with data collection repeated in a second and third round, each year for a total of three years. The main goals are to assess differences in outdoor activity patterns of participants with different levels of cognitive functioning and to analyze how participants' activity patterns are related to their own well-being and to that of their families [9].

To achieve the goals of the SenTra project, it is first critical to ascertain that elderly participants are able to comply with the research protocol. This is the focus of the current report. Measuring compliance in use of such technology is important as studies that have focused on other such communications, such as personal emergency response systems, have shown that some older individuals either do not wear the device at all or do not wear it consistently [10]. It is critical to assess the level of cooperation of research participants 
with the tracking protocol for both research and clinical purposes. For research purposes it is important to ensure that the behavior reported represents the actual behavior of the participant. This will be achieved when the participants are using the devices properly as described in the research protocol. For clinical purposes, compliance with the current study design will provide important information regarding the potential for participation in real life situations as this technology becomes more readily available.

Thus, the aim of the current study is to report on initial findings of such an assessment. As a preliminary step in the SenTra project, the Israeli team conducted a pilot study to assess the willingness and ability of participants to comply with a complex research protocol and to determine the degree to which participation was related to personal attributes of the participants and their families. Although the use of tracking technologies has become increasingly common in a variety of areas, to the best of our knowledge, this is the first systematic examination of factors related to participation of elderly participants in research involving such devices.

\section{METHODOLOGY}

\section{Participants}

Fifty-three participants aged $63+$ (24 male and 29 female) were recruited through the psycho-geriatric clinic at the Tel-Aviv Medical Center. Means and standard deviations of participants' background characteristics as well as all variables employed in the current study are found in Table $\mathbf{1}$. Participants belong to three groups: 18 unimpaired controls, 24 with mild cognitive impairment (MCI) and 11 with mild dementia. Participants with mild dementia were somewhat older $(\mathrm{M}=80.00, \mathrm{sd}=5.14)$ than healthy controls $[\mathrm{M}=72.44$, $\left.\mathrm{sd}=7.82 ; \chi^{2} \mathrm{~K}-\mathrm{w}=8.74, \mathrm{p}<.05\right]$ with participants with MCI between the two other groups $(M=75.29$, sd=5.52). There was no difference by sex between the three cognitive groups $\left[\chi^{2}(2)=1.14, \mathrm{p}>.05\right.$; healthy $50.00 \%$ male; MCI $37.50 \%$ male; mild dementia $54.55 \%$ male]. Participants with mild dementia had fewer years of education $(\mathrm{M}=11.73, \mathrm{sd}=4.02)$ than healthy controls $\left[\mathrm{M}=14.11, \mathrm{sd}=3.14 ; \chi_{\mathrm{K}-\mathrm{W}}^{2}=7.75, \mathrm{p}<.05\right]$ with participants with MCI not being significantly different than any of the other two groups ( $M=11.74$, sd=3.94).

Assignment to the three sample groups was based on the clinical diagnosis using DSM-4 operational criteria for dementia [11]. MCI criteria were based on Petersen et al. [12] and Winblad et al. [13] criteria. Screening included the Mini-Mental State Examination (MMSE) [14], NeuroPsychiatric Inventory (NPI) [15], and the Geriatric Depression Scale (GDS) [16]. Exclusion criteria included: MMSE score <21, severe motor and motility disorders (e.g. Parkinson disease), sensory impairment, certain medical problems, a history of substance abuse, major psychiatric illness or lack of Hebrew language skills. Elders residing in institutions were also excluded. Only elders with caregivers, potential caregivers or significant others living in close proximity, and willing to participate in the study as study partners were included.

Participants were tracked for four weeks (28 days). This time period was deemed long enough to characterize the regular activities of the elderly participants, yet not too long to be overwhelming and burdensome. All participants re-

Table 1. Mean, sd, and Range of Main Study Variables

\begin{tabular}{|c|c|c|}
\hline & Mean (sd) & Range \\
\hline Participant's age & $75.30(6.78)$ & $63-89$ \\
\hline Study partner's age & $60.37(14.35)$ & $24-81$ \\
\hline Study partner's education & $14.21(3.77)$ & $6-22$ \\
\hline GDS & $1.53(2.03)$ & $0-10$ \\
\hline NPI & $8.43(12.28)$ & $0-52$ \\
\hline Physical functioning & $75.83(22.94)$ & $10-100$ \\
\hline Role limitations due to physical state & $73.62(36.70)$ & $0-100$ \\
\hline General health & $58.49(16.37)$ & $15-85$ \\
\hline Positive affect & $30.49(7.04)$ & $15-45$ \\
\hline Negative affect & $17.51(5.65)$ & $9-32$ \\
\hline Life satisfaction & $7.81(1.83)$ & $3-10$ \\
\hline Caregiving burden & $8.68(7.51)$ & $0-33$ \\
\hline Caregiver's life satisfaction & $7.71(1.64)$ & $2-10$ \\
\hline
\end{tabular}


ceived a full explanation of the study's purpose and their roles, and were asked to identify a study partner (preferably a family member) who cared for them, or would care for them, if necessary. Study partners were recruited in this study for two reasons: First, given that the research protocol necessitated active participation of elders with cognitive impairments, the study partners' help was sought to facilitate and encourage participation. Further, study partners themselves were of direct interest in order to examine the influence of the use of the tracking technology on their quality of life.

Fifty-three family study partners participated in the study: 27 spouses of the participants; 24 adult children, one friend and one grandchild. All the spouses co-habitated with the participants, while the other study partners resided in separate households. Study partners were expected to know the participants well and have daily contact with them. Participants could withdraw from the study at any time. The study was approved by the IRB of the Tel Aviv Medical Center (NIH registration number NCT00743418).

\section{Instruments and Procedures}

Following diagnostic evaluation, participants and their study partners were interviewed at the participants' homes by licensed social workers, trained specially for this project.

The independent variables included: demographic variables, cognitive state, self-rated health and psycho-social variables. Demographic variables were: age, sex, years of education and study partner's relationship to the participant (spouse or other). Participant cognitive status was classified by major cognitive grouping as healthy, MCI or mild dementia. We also included mini-mental scores (from 21 to 30) and NPI scores (from 0 to 60).

Self-rated health was measured by six of the eight subscales of the SF-36 [17] questionnaire (physical functioning; role limitations due to physical health; role limitations due to emotional problems; social functioning; pain and general health). Each of the six sub-scales was transformed to range from 0 to 100 . The energy/fatigue and emotional well-being sub-scales were not used as they overlapped other questionnaires used in the study.
Emotional state of participants and study partners was assessed by the Positive and Negative Affect Schedule (PANAS) [18], in which each sub-scale ranged from 9 to 45 with higher scores indicating more positive and more negative affect. Life satisfaction was measured by a single item ranging from 0 (very unsatisfied) to 10 (very satisfied). In addition, study partners completed the short version of the Zarit Burden Interview [19], with scores ranging from 0 to 48 , with higher scores indicating higher degree of burden.

Following the interview, participants were provided with a location kit and instructed on its use. The kit was comprised of three elements (see Fig. 1): 1) a small portable unit containing a GPS receiver, GSM (Global System for Mobile Communication) modem and an RF (radio-frequency) receiver; 2) a wristwatch, that incorporates an RF transmitter and sensors that detect whether the watch is being worn on the body; and 3) a stationary home unit that repeats the RF signal and allows the participant to walk freely around the house without carrying the portable unit described above.

Participants were expected to wear the wristwatch at all times and take the portable unit with them whenever they left home all hours of the day for a period of 4 weeks. Study partners were expected to encourage and reassure the participant on the use of the tracking equipment by reminding the participant to take the device when needed; however, no clear guidelines were set for the study partners and each encouraged the participant according to their ability. Interviewers contacted participants and/ or their study partners by phone once a week with additional contacts if problems arose.

The RF transmitter-watch allows researchers to know whether participants are carrying the GPS device at any given moment. If the watchstrap is opened or the RF transmitter is not in contact with the body, the system notifies research staff. If the watch is worn on the hand, but is further than ten meters from the GPS receiver (at home, 70 meters because of the home monitoring unit) then a notification also will be issued. This combination of measures allowed researchers to ascertain the participant's level of participation at all times.
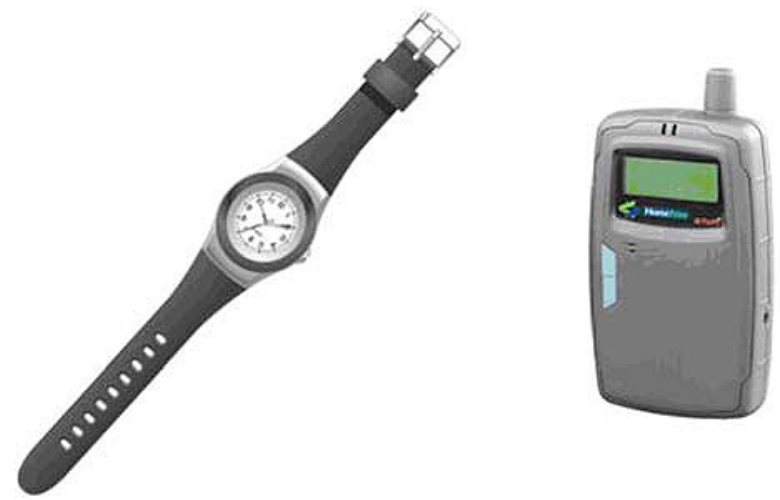

\section{Personal Watcher RF component}

\author{
STaR Monitoring Unit \\ GPS Receiver \\ GSM modem \\ RF component
}

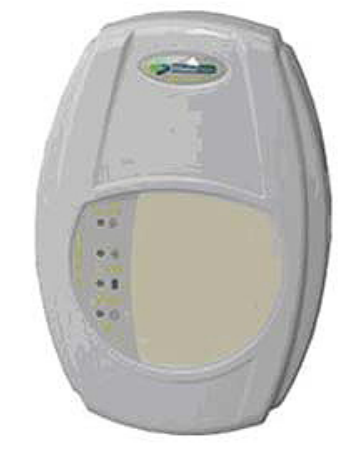
Home monitoring Unit RF component

Fig. (1). Elements of the location kit. 
When the participant is outside the home, the GPS receiver obtains locations every 10 seconds, for up to 12 hours without recharging. The data are sent by GPRS (General Packet Radio Service) to the project's server at the Hebrew University of Jerusalem, enabling researchers to monitor participation in real time. When problems related to the equipment or its use arose, interviewers contacted participants to review the protocol and resolve problems. Typical problems were related to the participant use; such as forgetting to charge the device or to take the device with them when leaving the home. The minority of the problems were related to the equipment, such as low battery strength on the watch or the GPS device. The current study is only concerned with problems related to the participants' use of the device.

In order to measure participation, a daily check was done for each participant for each hour of the previous day. Data obtained in a given hour were considered valid if researchers were certain as to the location of the participant. An hour was classified as having "valid" data if there were at least 30 minutes of valid data for that hour. On the other hand, if there were more than 30 minutes in which the watch was taken off the hand, or the GPS was not functioning (for reasons related to participant use), then the data for this hour were considered "not valid". Certain situations of missing data, which could be explained by the research participant, were included as valid in the analyses. For example, if a participant found it difficult to sleep wearing the watch or removed it while swimming and his location during the period was verified, these hours were considered "valid" for the analyses. Such situations were usually known to the interviewers during the initial interview.

Following four weeks of tracking, participants and study partners were re-interviewed using the same instruments detailed above. At this point, both participants and their study partners were asked to report on their satisfaction with the tracking device by employing an 8 item scale derived from the Quebec User Evaluation of Satisfaction with Assistive Technology [QUEST 2.0, [20]]. This scale rates satisfaction with the device's dimensions, watch's dimensions, device's weight and durability, ease of use, comfort, watch comfort and efficiency. A mean score of the eight items (ranging from 1 to 5) was calculated to represent satisfaction with the technology.

\section{Statistical Analyses}

Based upon the above criteria, two measures of cooperation were calculated for each participant: number of valid hours and percent of valid hours. Percent of valid hours was calculated by dividing the number of valid hours by the total number of hours tracked. The correlation between the num- ber of valid hours and the percent valid hours was high $\left[\mathrm{r}_{\mathrm{s}}(51)=0.83, \mathrm{p}<.001\right]$. These two dependent variables were then examined in relationship to the demographic, health, cognitive and psycho-social measures. Given the fairly small sample size, analyses are mainly descriptive and all were conducted using non-parametric statistics. Spearman correlations were used to determine the association between interval level independent variables and cooperation in the research. Mann-Whitney tests were applied for dichotomous independent variables while Kruksal-Wallis test was used for other categorical independent variables. Finally, a stepwise linear regression was conducted in which all variables found to be related to percent valid hours were entered into the regression equation.

\section{RESULTS}

Overall, the number of valid hours ranged from 232 to 691, with an average of 573.53 hours, sd $=93.10$. Percent valid hours in the entire sample ranged from $39.73 \%$ to $98.96 \%$, with an average of $84.39 \%$ (sd = 13.45). According to the Kolgomorov-Smirnov test, both number of valid hours and percent valid hours were normally distributed $(\mathrm{K}-\mathrm{S}=$ 1.14 and 1.21 respectively, p>.05). Table 2 shows the level of cooperation according to the three diagnosis groups. As seen, almost $70 \%$ of the participants had a high cooperation rate of more than $80 \%$ valid hours. Only two participants had a cooperation rate lower than $60 \%$ (specifically $40.28 \%$ and $39.73 \%$ ). Both of these were married female participants, one with MCI and one with mild dementia.

\section{Cooperation and Participant's Characteristics}

Table 3 shows the levels of cooperation by participants' and study partners' background variables. There was no significant difference in cooperation between male and female participants. Neither patient's age nor education were associated with cooperation. As to the study partners' characteristics, cooperation was not associated with study partners' gender; however number of valid hours was associated with study partners' age. However, participants with older study partners were more cooperative with the research protocol.

Table 4 shows the levels of cooperation by cognitive status and psychosocial state. In terms of cognitive status, the highest cooperation rate was found for MCI participants, followed by healthy and dementia participants. However, these differences were not significant. Cooperation was not related to participant depression as measured by the GDS (Geriatric Depression Scale) or to NPI scores. As for selfrated health, only physical functioning was found to be significantly correlated to cooperation according to percent valid hours.

Table 2. Levels of Participation by Cognitive Group

\begin{tabular}{|c|c|c|c|c|}
\hline & Healthy & MCI & Dementia & Overall \\
\hline \hline$\%$ Valid Hours & $\mathrm{N}(\%)$ & $\mathrm{N}(\%)$ & $\mathrm{N}(\%)$ & $2(9.09)$ \\
\hline $40-60 \%$ & 0 & $1(4.17)$ & $2(18.18)$ & $14(26.42)$ \\
\hline $61-80 \%$ & $8(44.44)$ & $4(16.67)$ & $8(72.70)$ & $37(69.81)$ \\
\hline $81-100 \%$ & $10(56.66)$ & $19(79.170)$ & & \\
\hline
\end{tabular}


Table 3. Levels of Participation by Participants' and Study Partners' Background Characteristics

\begin{tabular}{|c|c|c|}
\hline & $\begin{array}{l}\text { Valid Hours } \\
\text { Mean (sd) }\end{array}$ & $\begin{array}{c}\text { Percent Valid Hours } \\
\text { Mean (sd) }\end{array}$ \\
\hline \multicolumn{3}{|l|}{ Participant gender } \\
\hline Males & $583.71(71.73)$ & $85.21(10.88)$ \\
\hline Females & $565.10(108.21)$ & $83.71(15.42)$ \\
\hline Significance & $\mathrm{Z}=.00, \mathrm{p}>.05$ & $Z=-.14, p>.05$ \\
\hline \multicolumn{3}{|l|}{ Study partner gender } \\
\hline Males & $564.59(97.41)$ & $83.58(15.03)$ \\
\hline Females & $577.75(92.10)$ & $84.77(12.85)$ \\
\hline Significance & $Z=-.59, p>.05$ & $\mathrm{Z}=-.31, \mathrm{p}>.05$ \\
\hline \multicolumn{3}{|l|}{ Relationship to participant } \\
\hline Spouse & $592.67(62.33)$ & $87.41(9.60)$ \\
\hline Child & $550.17(118.91)$ & $80.37(16.50)$ \\
\hline \multirow[t]{2}{*}{ Significance } & $\mathrm{Z}=-.87, \mathrm{p}>.05$ & $Z=-1.48, p>.05$ \\
\hline & Correlation (R) & Correlation $(\mathrm{R})$ \\
\hline Participant's age & 0.07 & 0.06 \\
\hline Participant's education & -0.23 & -0.24 \\
\hline Study partner's age & $0.37 * *$ & $0.37 * *$ \\
\hline Study partner's education & $-0.28 *$ & -0.26 \\
\hline
\end{tabular}

$* \mathrm{p}<.05$.

$* * \mathrm{p}<.01$.

Table 4. Levels of Participation by Participants' and Study Partners' Cognitive and Psychosocial Characteristics

\begin{tabular}{|c|c|c|}
\hline & $\begin{array}{l}\text { Valid Hours } \\
\text { Mean (sd) }\end{array}$ & $\begin{array}{c}\text { \% Valid Hours } \\
\text { Mean (sd) }\end{array}$ \\
\hline \multicolumn{3}{|l|}{ Participant cognitive status } \\
\hline Healthy controls & $561.39(73.43)$ & $82.67(12.02)$ \\
\hline MCI & $588.71(98.73)$ & $86.51(13.16)$ \\
\hline Mild dementia & $560.27(111.71)$ & $82.58(16.65)$ \\
\hline \multirow[t]{2}{*}{ Significance } & $\chi_{\mathrm{K}-\mathrm{w}}^{2}=3.13, \mathrm{p}>.05$ & $\chi_{\mathrm{K}-\mathrm{w}}^{2}=1.68, \mathrm{p}>.05$ \\
\hline & Correlation $(\mathrm{R})$ & Correlation (R) \\
\hline MMSE & -0.06 & -0.06 \\
\hline GDS & -0.16 & -0.11 \\
\hline NPI & -0.02 & 0.08 \\
\hline \multicolumn{3}{|l|}{ Functional health } \\
\hline Physical functioning & -0.24 & $-0.29 *$ \\
\hline Role limitations due to physical state & -0.04 & -0.13 \\
\hline Role limitations due to emotional state & 0.02 & 0.12 \\
\hline Social functioning & -0.09 & -0.05 \\
\hline Pain & -0.11 & -0.09 \\
\hline General health & -0.08 & -0.13 \\
\hline Positive affect & $0.45 * * *$ & $0.32 *$ \\
\hline Negative affect & -0.05 & -0.21 \\
\hline Life satisfaction & $0.28 *$ & $0.34 *$ \\
\hline Caregiving burden & -0.17 & -0.28 \\
\hline
\end{tabular}

$* \mathrm{p}<.05 ; * * * \mathrm{p}<.001$. 
In terms of psycho-social variables, cooperation was found to be positively related to participants' positive affect and to their level of life satisfaction. Caregiver burden was not correlated with cooperation. Borderline significant differences were found between the three cognitive groups in the report of their satisfaction from the GPS device use $\left(\chi^{2} \mathrm{~K}\right.$ ${ }_{\mathrm{w}}=5.72, \mathrm{p}=.06$ with most satisfaction reported by participants with MCI, M=3.34, sd=.79; followed by participants with mild dementia, $\mathrm{M}=2.94$, $\mathrm{sd}=1.05$ and healthy controls $\mathrm{M}=2.68, \mathrm{sd}=1.02$ ).

Table 5 shows the results of a linear stepwise regression in which all variables that were found to be related to percent valid hours (participants' satisfaction from life and positive affect and caregivers' age) were entered into the equation. A stepwise regression was selected as one of its advantages over regular regression is that the former can substantially reduce the number of predictor variables to be used without losing a substantial portion of explanatory power of the data [21]. Results show that caregiver's age and participant's positive affect were both predictive of percent of valid hours; however, participant's life satisfaction no longer had a predictive capacity.

\section{DISCUSSION}

This study examined cooperation of cognitively impaired and non-impaired elderly persons with Global Positioning Systems-based research protocols and the relationship between cooperation and a variety of demographic, cognitive, health and psycho-social variables, based on a pilot study of 53 participants. Cooperation was found to be high. An average of $84 \%$ valid hours was noted indicating an ability to learn about the participant's out-of-home behavior during $84 \%$ of the hours of study.

None of the participants' demographic characteristics were found to be related to their level of cooperation. This indicates that elderly participants of different backgrounds are able to comply with the complex regimen of the current study over a fairly long period of time.

One explanation of the high levels of cooperation may be the strong bond formed between the participants and the interviewers over the course of the study. Previous research has shown that participation has been better when there is a positive relationship between patient and care provider [22]. This is particularly important in the current study in which interviewers were in frequent contact with the participants and were able to answer any of their questions regarding participation or try to find ways to help participants improve their cooperation (for example, place a note on the apartment door reminding them to take the equipment). The implications for research are that time and effort must be invested in the correct selection and training of interviewers so that they are able to form good relationships with study participants.

Another explanation of the high cooperation rate may be that participants view the technology as helpful for them. This is supported by anecdotal findings of requests by some participants to keep the GPS device with them after the study period has terminated. This request could not be granted at this point in the research as participants were expected to participate again the following year (in a repeated measure design). Also the device cannot be used for tracking without the use of the university website which was not possible for people not taking part in the research at a given time.

Although most of the participants' background characteristics were not related to their participation with the research design, their emotional wellbeing was related to their participation. Both higher life satisfaction scores and higher positive affect scores were related to more compliant behavior (although in the regression analysis only life satisfaction remained a significant predictor). One possible explanation for the better cooperation of participants with higher wellbeing may be related to personality traits which have been found to be related subjective well-being. Specifically, participation in this research may be viewed as an expression of "openness to experience" as the use of the GPS tracking device may be seen as an exciting venture. Openness to experience is a personality trait that is characterized by intelligence, culture, creativity, broad interests and cognitive complexity [23]. Openness to experience has been found to be related to happiness, positive affect, quality of life [24-26] and contentment [27]. An additional personality trait which might be explanatory is that of conscientiousness, that describes task behavior [23] and has also been found to relate to subjective wellbeing, especially life-satisfaction [24]. As the current research did not examine personality, this explanation requires further investigation.

Beyond the participants' characteristics, some of the study partners' background characteristics were found to be important for their relative's cooperation. Participants whose study partners were their spouses and were older were more likely to comply. This may reflect the spouses' encouragement for cooperation in the hope that electronic tracking would give them some peace of mind and eliminate future need to search for a lost relative [28].

The requirement for a study partner in research protocols may pose a threat to the participation of elderly persons in the study [29]. The current study points to the importance of careful selection of the study partner. The higher cooperation rate of participants whose study partners were their older spouses may be related to the geographic proximity which allowed the spouses, who all lived with the participants, to

Table 5. Stepwise Regression Analysis for Predicting Percent Valid Hours $(\mathbf{N}=53)$

\begin{tabular}{|c|c|c|c|c|c|}
\hline Predictors & $\boldsymbol{B}$ & SE B & Beta & $\boldsymbol{R}^{\mathbf{2}}$ & \multicolumn{2}{|c|}{$2^{* *}$} \\
\hline \hline & & & & 2.68 \\
\hline Participant positive affect & .64 & .24 & .33 & 2.89 & \\
\hline Caregiver's age & .33 & .12 & .35 & \\
\hline
\end{tabular}

$* * \mathrm{p}<.05$ 
encourage cooperation with the protocol. It is also possible that familial emotional proximity is of key importance. Spouses who are more involved with the participant than children, may be more aware of the difficulties in coping with a dementia patient in the home particularly in the early stages of decline [5] and may thus be a positive force in their study partners' continued participation.

The strength of this study is in showing that advanced tracking technologies offer a feasible method to measuring out-of-home mobility behavior in an elderly population, allowing researchers to obtain objective data from the elders themselves as opposed to observational reports of caregivers. However, although this study points to the ability to implement advanced tracking technologies within research, the daily use of such technologies raise many ethical issues and concerns; such as issues of privacy, autonomy and freedom. These ethical issues must be addressed before wide-spread use of these technologies among people with cognitive impairments is recommended. These ethical issues are being examined within the larger study [30].

The findings of this study have strong implications for the clinical application of GPS-based technologies. First, the high level of participation among participants of varying backgrounds may mean that elders in general may be able to utilize GPS devices for their everyday life needs. One of the study limitations is that the sample did not include any participants with severe forms of dementia and thus we can not raise any conclusions regarding the ability of this group to utilize the device. However, given the results we would suggest that use of GPS-based technologies should be employed during early stages of dementia. During these stages, the individual may learn to utilize and get adjusted to the device that may become inherent in their life. This hypothesis warrants further exploration.

The current findings point to the importance of the interviewers and the study partners in the use of the device. These significant others encouraged participants to comply with the study demands and reminded them to take the device when needed. These results may imply that elders who live on their own may find it more difficult to use such devices. Such elders may not have anyone available to remind them to charge the device and take the device with them. Further, these elders might be prone to loneliness and lower wellbeing which may also make it more difficult for them to utilize the device. Thus, programs and interventions might be developed that may help these individuals to benefit from such devices. For example, a reminder phone call in the morning and at nights.

The findings of this preliminary study must be considered in light of several limitations which also set the stage for future research. The main limitation of this study is its small sample size. Another issue is that the role of the study partners was not pre-determined. Strict guidelines may have changed the results of this study, possibly allowing for even better cooperation. Finally, all participants in the current study are community dwellers and none have severe dementia. It would be interesting to examine the use of these advanced technologies in additional settings such as adult day health centers, hospitals and long term care settings as well as among people with severe dementia. This would allow for the participation of participants at later stages of dementia.
Further, it would allow for comparison of study partners who are not family caregivers, but rather staff members at those settings.

\section{ACKNOWLEDGEMENTS}

Support of this project was provided by the German Federal Ministry of Education and Research (BMBF) within the framework of German-Israeli Project Cooperation (DIP).

\section{REFERENCES}

[1] Ferri CP, Prince M, Brayne C, et al. Global prevalence of dementia: A Delphi consensus study. Lancet 2005; 366: 2112-7.

[2] Mitchell L, Burton E, Raman S, Blackman T, Jenks M, Williams $\mathrm{K}$. Making the outside world dementia-friendly: Design issues and considerations. Environ Planning B: Planning Des 2003; 30: 60563.

[3] McShane R, Gedling K, Kenward B, Kenward R, Hope T, Jacoby $\mathrm{R}$. The feasibility of electronic tracking devised in dementia. Int $\mathbf{J}$ Geriatr Psychiatry 1998; 13: 556-63.

[4] Clyburn LD, Stones MJ, Hadjistavropoulos T, Tuokko H. Predicting caregiver burden and depression in Alzheimer's disease. J Gerontol B Psychol Sci Soc Sci 2000; 55: S2-13.

[5] Silverstein N, Flaherty G, Tobin TS. Dementia and wandering behavior: Concern for the lost elder. New York: Springer 2002.

[6] Logsdon RG, Teri L, McCurry SM, Gibbons LE, Kukull WA, Larson EB. Wandering: A significant problem among communityresiding individuals with Alzheimer's disease. J Gerontol B Psychol Sci Soc Sci 1998; 53: P294-9.

[7] Cohen-Mansfield J, Werner P, Marx MS, Freedman L. Two studies of pacing in the nursing home. J Gerontol 1991; 46: M77-83.

[8] Robinson L, Brittain K, Lindsay S, Jackson D, Oliver P. Keeping in touch everyday (KITE) project: Developing assistive technologies with people with dementia and their carers to promote independence. Int Psychogeriatr 2009; 21: 494-502.

[9] Shoval N, Auslander GK, Freytag T, et al. The use of advanced tracking technologies for the analysis of mobility in Alzheimer's disease and related cognitive diseases. BMC Geriatr 2008; 26: 7.

[10] Porter EJ. Wearing and using personal emergency response system buttons. J Gerontol Nurs 2005; 31: 26-33.

[11] American Psychiatric Association. Diagnostic and Statistical Manual of Mental Disorders. $4^{\text {th }}$ ed. Washington DC: American Psychiatric Association 1994

[12] Petersen RC. Mild cognitive impairment as a diagnostic entity. J Intern Med 2004; 256: 183-94.

[13] Winblad B, Palmer K, Kivipelto M, et al. Mild cognitive impairment-beyond controversies, towards a consensus: Report of the International Working Group on Mild Cognitive Impairment. J Int Med 2004; 256: 240-6.

[14] Folstein MF, Folstein SE, McHugh PR. "Mini-mental state". A practical method for grading the cognitive state of patients for the clinician. J Psychiatr Res 1975; 12: 189-98.

[15] Cummings JL, Mega M, Gray K, Rosenberg-Thompson S, Carusi DA, Gornbein J. The Neuropsychiatric Inventory: comprehensive assessment of psychopathology in dementia. Neurology 1994; 44: 2308-14.

[16] Yesavage JA, Brink TL, Rose TL, et al. Development and validation of a geriatric depression screening scale: a preliminary report. J Psychiatr Res 1982; 17: 37-49.

[17] Ware JE, Sherbourne CD. The MOS 36-item short-form health survey (SF-36). I. Conceptual framework and item selection. Med Care 1992; 30: 473-83

[18] Watson D, Clark LA, Carey G. Positive and negative affect and their relation to anxiety and depressive disorders. J Abnorm Psychol 1988; 97: 346-53.

[19] Bedard M, Molloy W, Squire L, Dubois S, Lever JA, O'Donnell M. The Zarit Burden Interview: A new short version and screening versions. Gerontologist 2001; 41: 652-7.

[20] Demers L, Weiss-Lambrou R, Ska B. Item analysis of the Quebec User Evaluation of Satisfaction with Assistive Technology (QUEST). Assist Technol 2000; 12: 96-105.

[21] Huang C, Townshend JRG. A stepwise regression tree for nonlinear approximation: Applications to estimating subpixel land cover. Int J Remote Sens 2003; 24: 75-90. 
[22] Okuno J, Yanagi H, Tomura S. Is cognitive impairment a risk factor for poor compliance among Japanese elderly in the community? Eur J Clin Pharmacol 2001; 57: 589-94.

[23] DeNeve KM, Cooper H. The happy personality: A meta-analysis of 137 personality traits and subjective well-being. Psychol Bull 1998; 124: $197-229$.

[24] Hayes N, Joseph S. Big 5 correlates of three measures of subjective well-being. Pers Individ Dif 2003; 34: 723-7.

[25] Gutierrez JLG, Jimenez BM, Hernandez EG, Puente CP. Personality and subjective well-being: Big five correlates and demographic variables. Pers Individ Dif 2005; 38: 1561-9.

[26] Steel P, Schmidt J, Shultz J. Refining the relationship between personality and subjective well-being. Psychol Bull 2008; 134: $138-61$.
[27] Mitte K, Kampfe N. Personality and the four faces of positive affect: A multitrait-multimethod analysis using self and peer report. J Res Pers 2008; 42: 1370-5.

[28] Robinson L, Hutchings D, Corner L, et al. Balancing rights and risks: Conflicting perspectives in the management of wandering in dementia. Health Risk Soc 2007; 9: 389-406.

[29] DeKosky ST. Maintaining adherence and retention in dementia prevention trials. Neurology 2006; 67: S14-6.

[30] Landau R, Werner S, Auslander GK, Shoval N, Heinik J. Attitudes of family and professional caregivers towards the use of GPS for tracking patients with dementia: An exploratory study. Br J Soc Work 2009; 39: 670-92.

Received: March 02, 2010

Revised: April 03, 2010

Accepted: June 12, 2010

(C) Auslander et al.; Licensee Bentham Open.

This is an open access article licensed under the terms of the Creative Commons Attribution Non-Commercial License (http://creativecommons.org/licenses/by-nc/3.0/) which permits unrestricted, non-commercial use, distribution and reproduction in any medium, provided the work is properly cited. 